\title{
Fecundity regulation in a spatial birth-and-death process
}

\author{
Viktor Bezborodov*1,2, Luca Di Persio ${ }^{\dagger 1}$, Dmitri Finkelshtein ${ }^{\ddagger 3}$, \\ Yuri Kondratiev ${ }^{\S 4}$, and Oleksandr Kutoviy ${ }^{\$ 4}$ \\ ${ }^{1}$ Department of Computer Science, The University of Verona, \\ Strada le Grazie 15, 37134 Verona, Italy \\ ${ }^{2}$ Faculty of Electronics, Wrocław University of Science and \\ Technology, Janiszewskiego 11/17, 50-372 Wrocław, Poland \\ ${ }^{3}$ Department of Mathematics, Swansea University, Bay Campus, \\ Fabian Way, Swansea SA1 8EN, U.K. \\ ${ }^{4}$ Fakultät für Mathematik, Universität Bielefeld, Postfach 110 131, \\ 33501 Bielefeld, Germany
}

September 30, 2019

\begin{abstract}
We study a Markov birth-and-death process on a space of locally finite configurations, which describes an ecological model with a density dependent fecundity regulation mechanism. We establish existence and uniqueness of this process and analyze its properties. In particular, we show global time-space boundedness of the population density and, using a constructed Foster-Lyapunov-type function, we study return times to certain level sets of tempered configurations. We find also sufficient conditions that the degenerate invariant distribution is unique for the considered process.
\end{abstract}

Mathematics subject classification: 60G55, 60J25, 82C21

Keywords: measure-valued process, Markov evolution, individual based models, spatial birth-and-death dynamics, spatial ecology, density dependent fecundity

\footnotetext{
*viktor . bezborodov@univr.it.

$\dagger$ luca.dipersio@univr.it.

${ }^{\ddagger}$ d.l.finkelshtein@swansea.ac.uk.

$\S_{\text {kondrat@math. uni-bielefeld.de. }}$

"kutoviy@math. uni-bielefeld.de.
} 


\section{Introduction}

Most of models in ecology are structured by space. Nowadays, individual based models in spatial ecology form a well established research area. We refer for historical comments and detailed review to [25]. Mathematically, such models may be often described as Markov birth-and-death processes on configuration spaces over proper location sets. A simple example is an independent birth process in a population $\eta$ located in the Euclidean space $\mathbb{R}^{\mathrm{d}}$ : each member of the population $\eta$, placed at $y \in \eta$, independently sends its off-spring to the target location $x \in \mathbb{R}^{\mathrm{d}}$ after an exponentially distributed random time. The displacement $x-y$ is chosen at random according to a certain dispersion kernel. The rate $\varkappa>0$ of the time-distribution is called the (density independent) fecundity rate. Then, regardless of a particular dispersion kernel, the density of the population will grow exponentially in time.

The simplest regulation mechanism to prevent the unbounded growth is to include a (density independent) mortality to the process. Namely, each member of the population may die after an exponentially distributed random time (independent from the birth time) with a rate $m>0$. Then, for $m<\varkappa$, the density of the system is still exponentially increasing in time, whereas $m=\varkappa$ is a critical value where the density is stabilized, and finally, for $m>\varkappa$, the density will exponentially decay showing the extinction of the population. This process can be treated also as a nonlocal branching process (cf. e.g. [22, Section 4.3]), namely, each member dies after random time and, with certain rates, may produce 0 or 2 off-springs with the restriction that one of the latter is placed at parent's position, see [2]. We will call this process spatial contact model following [21], see also [18,20]. Note that, in the so-called critical case $m=\varkappa$, the considered system will have a unique invariant distribution (a probability measure over the space of configurations in $\mathbb{R}^{\mathrm{d}}$ ) for each initial mean density, in any dimension $d \geq 3$. This measure, in particular, has fast growth of factorial moments.

A more sophisticated regulation mechanism is to consider a density dependent mortality rate. Such a rate is just the sum of the constant mortality and competitions with all other members of the population defined through a competition kernel. This describes the so-called spatial logistic model, see [25] and references therein for its biological motivations. The corresponding Markov processes on finite sets (populations) in $\mathbb{R}^{\mathrm{d}}$ was analysed in [12], see also [3]. Infinite populations were studied mathematically in $[6,7]$ and $[16,17]$ in terms of the evolution of states (measures) on the space of locally finite configurations. Such approach to study dynamics of infinite populations is known as statistical one. More precisely, the evolution of states in this approach is described by the evolution of the corresponding factorial moments (a.k.a. correlation functions). Note that a construction of the corresponding Markov process remains a challenging problem. 
To the best of our knowledge, for locally finite systems in $\mathbb{R}^{\mathrm{d}}$, there are only few references concerning the construction of a general Markov birthand-death process, namely, $[1,13,14]$. In the present paper we study several models which belong to the considered in [14] case with a constant death rate and a birth rate with certain structural properties (see Section 2 below for details); the process is constructed as a solution to stochastic equation. On the contrary, a birth-and-death process with constant birth rate and the aforementioned density dependent mortality was constructed in [1] using a different approach which is based on a comparison with a Poisson random connection graph.

The main novelty of the present paper is that we consider a rather different regulation mechanism compared to the spatial logistic model. Namely, keeping a constant (density independent) mortality rate, we consider birth with a density dependent fecundity of the form

$$
\varkappa(y, \eta):=\varkappa e^{-l(y, \eta)}, \quad l(y, \eta):=\sum_{z \in \eta \backslash\{y\}} \varphi(y-z),
$$

where, we recall, $\eta \subset \mathbb{R}^{\mathrm{d}}$ represents the locally finite set of positions of the population members. In other words, the competition (described by the kernel $\varphi$ ) within the population does not influence chances to die, but decreases chances of producing off-springs. We show (Proposition 3.2) that under minimal restrictions on the dispersion and the competition kernels the whole birth rate for the system (which includes summation over different $y \in \eta$ ) remains globally bounded as a function of the existing configuration and the position for an off-spring. This allows to show the existence and uniqueness of the corresponding process (Theorem 3.3). It is worth noting that we do not require any comparison between the dispersion and the competition kernels. We allow also a modification of (1.1) with (in the simplest model case)

$$
\varkappa(y, \eta)=\varkappa e^{-l(y, \eta)}(1+p l(y, \eta)), \quad p \geq 0 .
$$

This modification is well motivated biologically in the case $p>1$ as then the competition starts to affect negatively the sending of off-springs only after the population becomes 'well-developed' (i.e. the value of $s:=l(y, \eta)$ becomes large enough and the unimodal function $\varkappa e^{-s}(1+p s)$ starts to decay). This corresponds to the so-called weak Allee effect, see e.g. [27]. Note that such generalization was considered also in [8] in terms of the aforementioned statistical dynamics; the existence and properties of the corresponding Markov process remained open since then.

In Section 4, we study some general properties of processes with constant death and bounded birth rates (in addition to the fecundity model above, we consider two others, see Examples 4.1-4.2). In particular, we prove that return times to sufficiently large level sets of configurations are exponentially integrable random variables (Proposition 4.15). To show this, we introduce 
and study a Forster-Lyapunov function on the space of (tempered) configurations.

Finally, in Section 5, we introduce sufficient conditions for general sublinear birth rates (including, in particular, those for the fecundity model), which ensure the uniqueness of the degenerate invariant distribution for the considered process.

\section{Birth and death processes on configuration space}

Let $\mathscr{B}\left(\mathbb{R}^{\mathrm{d}}\right)$ denote the Borel $\sigma$-algebra over the Euclidean space $\mathbb{R}^{\mathrm{d}}, \mathrm{d} \in \mathbb{N}$. We study a birth-and-death process taking values in the space of locally finite configurations (discrete subsets) of $\mathbb{R}^{\mathrm{d}}$ :

$$
\Gamma:=\left\{\eta \subset \Lambda|| \eta \cap B \mid<+\infty \text { for any bounded } B \in \mathscr{B}\left(\mathbb{R}^{\mathrm{d}}\right)\right\} .
$$

Henceforth, $|\eta|$ denotes the number of points in a discrete finite set $\eta \subset \mathbb{R}^{\mathrm{d}}$.

Throughout the paper, we identify a configuration $\eta \in \Gamma$ with a discrete (counting) measure on $\left(\mathbb{R}^{\mathrm{d}}, \mathscr{B}\left(\mathbb{R}^{\mathrm{d}}\right)\right)$ defined by assigning a unit mass to each atom at $x \in \eta$.

We fix an arbitrary $\varepsilon>0$ and consider the function

$$
G(x):=(1+|x|)^{-\mathrm{d}-\varepsilon}, \quad x \in \mathbb{R}^{\mathrm{d}},
$$

where $|x|$ denotes the Euclidean norm on $\mathbb{R}^{\mathrm{d}}$. We denote then

$$
\Gamma_{G}:=\left\{\eta \in \Gamma \mid\langle G, \eta\rangle:=\sum_{x \in \eta} G(x)<\infty\right\},
$$

a set of tempered configurations. We define a sequential topology on $\Gamma_{G}$ by assuming that $\eta_{n} \rightarrow \eta, n \rightarrow \infty$, if only

$$
\lim _{n \rightarrow \infty}\left\langle f, \eta_{n}\right\rangle=\langle f, \eta\rangle
$$

for all $f \in C_{b}\left(\mathbb{R}^{\mathrm{d}}\right)$ (the space of bounded continuous functions on $\mathbb{R}^{\mathrm{d}}$ ) such that $|f(x)| \leq M_{f} G(x)$ for some $M_{f}>0$ and all $x \in \mathbb{R}^{\mathrm{d}}$. Let $\mathscr{B}_{G}(\Gamma)$ denote the corresponding Borel $\sigma$-algebra.

Let $b: \mathbb{R}^{\mathrm{d}} \times \Gamma_{G} \rightarrow \mathbb{R}_{+}:=[0, \infty)$ be a measurable function. We describe a spatial birth-and-death process $\eta: \mathbb{R}_{+} \rightarrow \Gamma_{G}$ with the unit death rate and the birth rate $b$ through the following three properties:

1. If the system is in a state $\eta_{t} \in \Gamma_{G}$ at the time $t \in \mathbb{R}_{+}$, then the probability that a new particle appears (a "birth" happens) in a bounded set $B \in \mathscr{B}\left(\mathbb{R}^{\mathrm{d}}\right)$ during a time interval $[t ; t+\Delta t]$ is

$$
\Delta t \int_{B} b\left(x, \eta_{t}\right) d x+o(\Delta t) .
$$


2. If the system is in a state $\eta_{t} \in \Gamma_{G}$ at the time $t \in \mathbb{R}_{+}$, then, for each $x \in \eta_{t}$, the probability that the particle at $x$ dies during a time interval $[t ; t+\Delta t]$ is $1 \cdot \Delta t+o(\Delta t)$.

3. With probability 1 no two events described above happen simultaneously.

The (heuristic) generator of our process is

$$
L F(\eta)=\sum_{x \in \eta}(F(\eta \backslash\{x\})-F(\eta))+\int_{\mathbb{R}^{\mathrm{d}}} b(x, \eta)(F(\eta \cup\{x\})-F(\eta)) d x .
$$

Definition 2.1. Let a right-continuous complete filtration $\left\{\mathcal{F}_{t}\right\}$ be fixed.

1. Let $N$ be the Poisson point process on $\mathbb{R}_{+} \times \mathbb{R}^{\mathrm{d}} \times \mathbb{R}_{+}^{2}$ with mean measure $d s \times d x \times d u \times e^{-r} d r$, compatible w.r.t. the filtration $\left\{\mathcal{F}_{t}\right\}$. The latter means that for any measurable $A \subset \mathbb{R}^{\mathrm{d}} \times \mathbb{R}_{+}^{2}, N([0, t], A)$ is $\mathcal{F}_{t}$-measurable and $N((t, s], A)$ is independent of $\mathcal{F}_{t}$ for $0<t<s$.

2. Let $\eta_{0}$ be a $\Gamma_{G}$-valued $\mathcal{F}_{0}$-measurable random variable independent on $N$. Consider a point process $\tilde{\eta}_{0}$ on $\mathbb{R}^{\mathrm{d}} \times \mathbb{R}_{+}$obtained by attaching to each point of $\eta_{0}$ an independent unit exponential random variable. Namely, if $\eta_{0}=\left\{x_{i}: i \in \mathbb{N}\right\}$ then $\tilde{\eta}_{0}=\left\{\left(x_{i}, \tau_{i}\right): i \in \mathbb{N}\right\}$ and $\left\{\tau_{i}\right\}$ are independent unit exponentials, independent of $\eta_{0}$ and $N$.

3. We will say that a process $\left(\eta_{t}\right)_{t \geq 0}$ with sample paths in the Skorokhod space $D_{\Gamma_{G}}[0, \infty)$ has the unit death rate and the birth rate $b$ if it is adapted to a filtration $\left\{\mathcal{F}_{t}\right\}$ w.r.t. to which $N$ is compatible and if, for any bounded $B \in \mathscr{B}\left(\mathbb{R}^{\mathrm{d}}\right)$, the following equality holds almost surely

$$
\begin{aligned}
\eta_{t}(B)= & \int_{(0, t] \times B \times \mathbb{R}_{+}^{2}} I_{\left[0, b\left(x, \eta_{s-}\right)\right]}(u) I_{[t-s, \infty)}(r) N(d s, d x, d u, d r) \\
& +\int_{B \times \mathbb{R}_{+}} I_{[t, \infty)}(r) \tilde{\eta}_{0}(d x, d r)
\end{aligned}
$$

where $\eta_{t}(B)=\left|\eta_{t} \cap B\right|$ is the number of points in $B$; note that henceforth we use configurations and counting measures interchangeably.

Remark 2.2. Following a convention for continuous-space processes, see e.g. [14], we will say that the function $b$ is the birth rate of the process, even though the rate of birth inside a bounded region $B \in \mathscr{B}\left(\mathbb{R}^{\mathrm{d}}\right)$ is given by

$$
\nu_{\eta}(B):=\int_{B} b(x, \eta) d x,
$$

that is, $\mathbb{P}\left\{\left|\left(\eta_{t} \backslash \eta_{0}\right) \cap B\right|=1\right\}=\nu_{\eta_{0}}(B) t+o(t)$. Thus, the function $b$ is actually a version of the Radon-Nikodym derivative of the rate (considered 
as the measure $\nu_{\eta}$ ) w.r.t. the Lebesgue measure. For the notion of the transition rates for interacting particle systems, see e.g. [23, Chapter 1.3].

Remark 2.3. In (2.4), $x$ is a location of a particle from $\left(\eta_{t}\right)_{t \geq 0}, s$ is the birth time of the particle (equals 0 if $x \in \eta_{0}$ ), $u$ is the randomizing variable deciding whether the birth actually occurs, and finally $r$ is a lifespan of the particle. The constant death rate in our model means that each particle has an exponentially distributed lifetime, which explains the presence of the multiplier $e^{-r}$ in the density of $N$.

Remark 2.4. We will sometimes denote the solution process at time $t$ as $\eta\left(t, \eta_{0}\right)$ to underline the dependence on the initial condition. In other words, $\eta_{t}=\eta\left(t, \eta_{0}\right)$. As is the convention for Markov processes, we use notation $\mathbb{E}_{\eta_{0}}$ for the expectation related to the distribution of $\left(\eta\left(t, \eta_{0}\right)\right)_{t \geq 0}$.

Definition 2.5. Let $\Gamma \ni \eta=\left\{x_{i}\right\}$ and $y \in \mathbb{R}^{\mathrm{d}}$. We set $S_{y} \eta:=\left\{x_{i}-y\right\} \in \Gamma$.

1. The birth rate $b$ is said to be translation invariant if

$$
b(x+y, \eta)=b\left(x, S_{y} \eta\right), \quad x \in \mathbb{R}^{\mathrm{d}}, \eta \in \Gamma_{G} .
$$

2. A $\Gamma_{G}$-valued random variable $\eta$ is said to be translation invariant if the distribution of $S_{y} \eta$ does not depend on $y$.

The following statement is a particular case of results from [14].

Theorem 2.6. Suppose that

$$
\mathbf{b}:=\sup _{\substack{x \in \mathbb{R}^{\mathrm{d}} \\ \eta \in \Gamma_{G}}} b(x, \eta)<\infty
$$

and, for some $M>0$,

$$
\sup _{\eta \in \Gamma_{G}}|b(x, \eta \cup\{y\})-b(x, \eta)| \leq M G(x-y), \quad x, y \in \mathbb{R}^{\mathrm{d}} .
$$

Then there exists a pathwise unique solution to (2.4) in the sense of Definition 2.1. If, additionally, both $b$ and $\eta_{0}$ are translation invariant, then $\eta_{t}$ is translation invariant for $t>0$.

To prove the latter statement, we need the following simple lemma.

Lemma 2.7. For any $x, y, z \in \mathbb{R}^{\mathrm{d}}$,

$$
G(x-y) G(y-z) \leq G(x-z) .
$$

Proof. Immediately follows from (2.1) and the inequality

$$
1+|x-z| \leq 1+|x-y|+|y-z| \leq(1+|x-y|)(1+|y-z|) .
$$


Proof of Theorem 2.6. We follow the ideas of [26, Remark 4.1]. First of all, (2.5) yields

$$
\sup _{\eta \in \Gamma_{G}} \int_{\mathbb{R}^{\mathrm{d}}} G(x) b(x, \eta) d x \leq \mathbf{b} \int_{\mathbb{R}^{\mathrm{d}}} G(x) d x<\infty,
$$

that implies that $[14$, Condition 2.1] is satisfied.

Next, by [14, Lemma 2.15], (2.6) implies that, for any $\eta_{1}, \eta_{2} \in \Gamma_{G}$ and $x \in \mathbb{R}^{\mathrm{d}}$,

$$
\left|b\left(x, \eta_{1}\right)-b\left(x, \eta_{2}\right)\right| \leq M \int_{\mathbb{R}^{\mathrm{d}}} G(x-y)\left|\eta_{1}-\eta_{2}\right|(d y),
$$

where $\left|\eta_{1}-\eta_{2}\right|$ means the total variation norm of the (signed) discrete measure $\eta_{1}-\eta_{2}$ on $\left(\mathbb{R}^{\mathrm{d}}, \mathscr{B}\left(\mathbb{R}^{\mathrm{d}}\right)\right.$ ). By using (2.7) with $z=0$ (and swapping $x$ and $y$ ), we get that, for each $x \in \mathbb{R}^{\mathrm{d}}$,

$$
\left|b\left(x, \eta_{1}\right)-b\left(x, \eta_{2}\right)\right| \leq M(1+|x|)^{d+\varepsilon} \int_{\mathbb{R}^{\mathrm{d}}} G(y)\left|\eta_{1}-\eta_{2}\right|(d y),
$$

that implies that [14, Condition 2.2] is satisfied.

The last assumption of [14, Theorem 2.13] can be read in our settings as follows

$$
\sup _{x} \int_{\mathbb{R}^{\mathrm{d}}} \frac{c(x) M G(x-y)}{c(y)} d y<\infty
$$

for some positive bounded (cf. [26, Remarks 4.1(a)]) function $c$; the latter inequality evidently holds with $c \equiv 1$. As a result, one gets the statement from [14, Theorem 2.13, Lemma 3.14].

\section{Description of the model}

We consider a birth-and-death process on $\Gamma_{G}$ with the unit death rate and the birth rate given, for some integrable functions $a, c, \varphi: \mathbb{R}^{\mathrm{d}} \rightarrow \mathbb{R}_{+}$, by

$$
b(x, \eta)=\sum_{y \in \eta} a(x-y)\left(1+\sum_{z \in \eta \backslash\{y\}} c(z-y)\right) \exp \left(-\sum_{z \in \eta \backslash\{y\}} \varphi(z-y)\right) .
$$

In view of (2.2), the rate (3.1) can be interpreted as follows. Let $\varkappa=$ $\int_{\mathbb{R}^{\mathrm{d}}} a(x) d x>0$. If the system is in a state $\eta \in \Gamma_{G}$ at the time $t \in \mathbb{R}_{+}$, then each $y \in \eta$ may send an off-spring after exponential random time whose rate is $\varkappa r(y, \eta)$, where

$$
r(y, \eta):=\left(1+\sum_{z \in \eta \backslash\{y\}} c(z-y)\right) \exp \left(-\sum_{z \in \eta \backslash\{y\}} \varphi(z-y)\right) .
$$

The off-spring will be sent according to the probability distribution on $\mathbb{R}^{\mathrm{d}}$ with the (normalized) density $\varkappa^{-1} a$, i.e. the probability that the off-spring 
(sent from $\left.y \in \eta_{t}\right)$ appears in a bounded $B \in \mathscr{B}\left(\mathbb{R}^{\mathrm{d}}\right)$ is $\varkappa^{-1} \int_{B} a(x-y) d x$. Note that we allow $c=0$ a.e.

In ecology, the rate $\varkappa r(y, \eta)$ is called the fecundity. A model example is the case when $c(z)=p \varphi(z), z \in \mathbb{R}^{\mathrm{d}}$, for some $p \geq 0$. Then (3.2) is just the value of $R_{p}(s):=(1+p s) e^{-s}, s \in \mathbb{R}_{+}$, at $s=\sum_{z \in \eta \backslash\{y\}} \varphi(z-y)$. The function $R_{p}$ is decreasing on $\mathbb{R}_{+}$for $p \in[0,1]$ and is unimodal for $p>1$, i.e. it has a unique maximum point; at $s=\frac{p-1}{p}$. As a result, the case $p \in[0,1]$ describes the model where the 'wish' for $y \in \eta_{t}$ to send an off-spring decays because of other particles around $y$. Whereas the case $p>1$ describes the so-called weak Allee effect when the small density of the system around $y$ increases the chances for an off-spring to be sent, but there exists a threshold for that density after which the surrounders of $y$ decrease the chances.

The following lemma is the key tool in proving the global boundedness of the birth rate (3.1).

Lemma 3.1. Let $\alpha, \rho>0$, and let $b_{f}: \mathbb{R}_{+} \rightarrow(0, \infty)$ be a decreasing function, convergent to 0 at $\infty$, such that

$$
\int_{\mathbb{R}_{+}} b_{f}(s) s^{\mathrm{d}-1} d s<\infty .
$$

Let $f, g: \mathbb{R}^{\mathrm{d}} \rightarrow \mathbb{R}_{+}$be measurable functions, $f$ is bounded, such that

$$
\begin{array}{ll}
f(x) \leq b_{f}(|x|), & x \in \mathbb{R}^{\mathrm{d}}, \\
g(x) \geq \alpha, & |x| \leq \rho .
\end{array}
$$

Then

$$
\sup _{\substack{x \in \mathbb{R}^{\mathrm{d}} \\ \eta \in \Gamma}} \sum_{y \in \eta} f(x-y) \exp \left\{-\sum_{z \in \eta \backslash\{y\}} g(z-y)\right\}<\infty .
$$

Proof. For each $x=\left(x_{1}, \ldots, x_{d}\right) \in \mathbb{R}^{\mathrm{d}}$, let $|x|$ denote the Euclidean norm of $x$, and let $|x|_{\infty}:=\max _{1 \leq i \leq \mathrm{d}}\left|x_{i}\right|$. We have then

$$
|x|_{\infty} \leq|x| \leq \sqrt{\mathrm{d}}|x|_{\infty}, \quad x \in \mathbb{R}^{\mathrm{d}} .
$$

Set $q:=\frac{\rho}{\sqrt{\mathrm{d}}}>0$, and, for each $\boldsymbol{k}=\left(k_{1}, \ldots, k_{d}\right) \in \mathbb{Z}^{\mathrm{d}}$, consider a cube

$$
H_{q}(\boldsymbol{k})=\left\{x \in \mathbb{R}^{\mathrm{d}} \mid q\left(k_{i}-\frac{1}{2}\right)<x_{k} \leq q\left(k_{i}+\frac{1}{2}\right)\right\}
$$

centered at $q \boldsymbol{k} \in \mathbb{R}^{\mathrm{d}}$ with edges of the length $q$. Note that $\mathbb{R}^{\mathrm{d}}=\bigsqcup_{\boldsymbol{k} \in \mathbb{Z}^{\mathrm{d}}} H_{q}(\boldsymbol{k})$.

For each $z \in \mathbb{R}^{\mathrm{d}}$ with $|z|_{\infty} \leq q$, we have, by (3.6), $|z| \leq \rho$, and hence, by (3.5), $g(z) \geq \alpha$. Therefore, for $x, y \in H_{q}(\boldsymbol{k}), \boldsymbol{k} \in \mathbb{Z}^{\mathrm{d}}$, we have $|x-y|_{\infty} \leq q$ and hence

$$
g(x-y) \geq \alpha, \quad x, y \in H_{q}(\boldsymbol{k}), \boldsymbol{k} \in \mathbb{Z}^{\mathrm{d}} .
$$


Furthermore, for $x \in H_{q}\left(\boldsymbol{k}_{x}\right), y \in H_{q}\left(\boldsymbol{k}_{y}\right), \boldsymbol{k}_{x}, \boldsymbol{k}_{y} \in \mathbb{Z}^{\mathrm{d}}$, we have $\left|x-q \boldsymbol{k}_{x}\right| \leq \frac{\rho}{2}$, $\left|y-q \boldsymbol{k}_{y}\right| \leq \frac{\rho}{2}$, and

$$
|x-y| \geq-\left|x-q \boldsymbol{k}_{x}\right|+q\left|\boldsymbol{k}_{x}-\boldsymbol{k}_{y}\right|-\left|q \boldsymbol{k}_{y}-y\right| \geq q\left|\boldsymbol{k}_{x}-\boldsymbol{k}_{y}\right|-\rho,
$$

and hence by (3.4) and the monotonicity of $b_{f}$

$$
f(x-y) \leq b_{f}(|x-y|) \leq b_{f}\left(\left(q\left|\boldsymbol{k}_{x}-\boldsymbol{k}_{y}\right|-\rho\right) \vee 0\right) .
$$

As a result, for any $\eta \in \Gamma$,

$$
\begin{gathered}
\sum_{y \in \eta} \exp \left(-\sum_{z \in \eta \backslash\{y\}} g(z-y)\right) f(x-y) \\
=\sum_{\boldsymbol{k} \in \mathbb{Z}^{\mathrm{d}}} \sum_{y \in \eta \cap H_{q}(\boldsymbol{k})} \exp \left(-\sum_{\boldsymbol{j} \in \mathbb{Z}^{\mathrm{d}}} \sum_{z \in\left(\eta \cap H_{q}(\boldsymbol{j})\right) \backslash\{y\}} g(z-y)\right) f(x-y) \\
\leq \sum_{\boldsymbol{k} \in \mathbb{Z}^{\mathrm{d}}} \sum_{y \in \eta \cap H_{q}(\boldsymbol{k})} \exp \left(-\sum_{z \in(\eta \backslash\{y\}) \cap H_{q}(\boldsymbol{k})} g(z-y)\right) f(x-y) \\
\leq \sum_{\boldsymbol{k} \in \mathbb{Z}^{\mathrm{d}}} \sum_{y \in \eta \cap H_{q}(\boldsymbol{k})} \exp \left(-\alpha\left|(\eta \backslash\{y\}) \cap H_{q}(\boldsymbol{k})\right|\right) b_{f}\left(\left(q\left|\boldsymbol{k}-\boldsymbol{k}_{x}\right|-\rho\right) \vee 0\right) \\
=\sum_{\boldsymbol{k} \in \mathbb{Z}^{\mathrm{d}}}\left|\eta \cap H_{q}(\boldsymbol{k})\right| \exp \left(-\alpha\left|\eta \cap H_{q}(\boldsymbol{k})\right|\right) e^{\alpha} b_{f}\left(\left(q\left|\boldsymbol{k}-\boldsymbol{k}_{x}\right|-\rho\right) \vee 0\right) \\
\leq \frac{e^{\alpha}}{\alpha e} \sum_{\boldsymbol{k} \in \mathbb{Z}^{\mathrm{d}}} b_{f}\left(\left(q\left|\boldsymbol{k}-\boldsymbol{k}_{x}\right|-\rho\right) \vee 0\right)=\frac{e^{-\alpha}}{\alpha e} \sum_{\boldsymbol{j} \in \mathbb{Z}^{\mathrm{d}}} b_{f}((q|\boldsymbol{j}|-\rho) \vee 0)<\infty,
\end{gathered}
$$

because of (3.3), and the bound does not depend on $x$. In the penultimate inequality we used that $s e^{-\alpha s} \leq \frac{1}{\alpha e}$ for $s \geq 0$. The lemma is proved.

To show the existence of the process (see Theorem 3.3 below), we will require the following assumptions.

Condition 1. There exists $B \geq 1$, such that, for $x \in \mathbb{R}^{\mathrm{d}}$,

$$
a(x) \leq B G^{2}(x), \quad \varphi(x) \leq B G(x) .
$$

Condition 2. The function $\varphi$ is separated from 0 in a neighborhood of the origin.

Condition 3. There exists $p \geq 0$, such that $c(x) \leq p \varphi(x)$ for $x \in \mathbb{R}^{\mathrm{d}}$.

Proposition 3.2. Let the birth rate $b$ is given by (3.1). Suppose that there exists $B \geq 1$ such that, for $x \in \mathbb{R}^{\mathrm{d}}$,

$$
a(x) \leq B G(x), \quad \varphi(x) \leq B G(x)
$$

(in particular, let Conditions 1 hold). Suppose also that Conditions 2-3 hold. Then $b$ is uniformly bounded, i.e. (2.5) holds. 
Proof. Condition 3 implies that

$$
\begin{aligned}
b(x, \eta) \leq & \sum_{y \in \eta} a(x-y) \exp \left(-\frac{1}{2} \sum_{z \in \eta \backslash\{y\}} \varphi(z-y)\right) \\
& \times\left(1+p \sum_{z \in \eta \backslash\{y\}} \varphi(z-y)\right) \exp \left(-\frac{1}{2} \sum_{z \in \eta \backslash\{y\}} \varphi(z-y)\right) \\
\leq & C_{p} \sum_{y \in \eta} a(x-y) \exp \left(-\frac{1}{2} \sum_{z \in \eta \backslash\{y\}} \varphi(z-y)\right)
\end{aligned}
$$

where

$$
C_{p}=\sup _{s \geq 0}(1+p s) e^{-\frac{1}{2} s}= \begin{cases}1, & 0 \leq p \leq \frac{1}{2} \\ 2 p e^{\frac{1}{2 p}-1}, & p>\frac{1}{2}\end{cases}
$$

By Condition 2 and the first inequality in (3.7), one can apply Lemma 3.1 with $f=a, g=\frac{1}{2} \varphi, b_{f}(s)=B(1+s)^{-\mathrm{d}-\varepsilon}$ to (3.8), that yields the statement.

Theorem 3.3. Let $b$ be the birth rate given by (3.1) and Conditions 1-3 hold. Then there exists a unique solution to (2.4) in the sense of Definition 2.1. If, additionally, both $b$ and $\eta_{0}$ are translation invariant, then $\eta_{t}$ is translation invariant for $t>0$.

Proof. We apply Theorem 2.6. Since Proposition 3.2 implies (2.5), it is enough to check that (2.6) holds. For $\eta \in \Gamma_{G}, x \in \mathbb{R}^{\mathrm{d}}$ and $x^{\prime} \in \mathbb{R}^{\mathrm{d}} \backslash\{x\}$, we have from (3.1) and (3.2),

$$
\begin{aligned}
& b\left(x, \eta \cup\left\{x^{\prime}\right\}\right)-b(x, \eta) \\
= & \sum_{y \in \eta} a(x-y) e^{-\varphi\left(x^{\prime}-y\right)} r(y, \eta) \\
+ & \sum_{y \in \eta} a(x-y) c\left(x^{\prime}-y\right) e^{-\varphi\left(x^{\prime}-y\right)} \exp \left(-\sum_{z \in \eta \backslash\{y\}} \varphi(z-y)\right) \\
& +a\left(x-x^{\prime}\right) r\left(x^{\prime}, \eta\right)-\sum_{y \in \eta} a(x-y) r(y, \eta) .
\end{aligned}
$$

Then, by using inequalities $\left|e^{-\varphi}-1\right| \leq \varphi$ and $0 \leq c \leq p \varphi$, we get

$$
\begin{aligned}
& \left|b\left(x, \eta \cup\left\{x^{\prime}\right\}\right)-b(x, \eta)\right| \\
\leq & \sum_{y \in \eta} a(x-y) \varphi\left(x^{\prime}-y\right)(1+p) r(y, \eta) \\
& +a\left(x-x^{\prime}\right)\left(1+p \sum_{z \in \eta} \varphi\left(z-x^{\prime}\right)\right) \exp \left(-\sum_{z \in \eta} \varphi\left(z-x^{\prime}\right)\right) .
\end{aligned}
$$


Next, by (3.7) and (2.7),

$$
a(x-y) \varphi\left(x^{\prime}-y\right) \leq B^{2} G^{2}(x-y) G\left(x^{\prime}-y\right) \leq B^{2} G(x-y) G\left(x-x^{\prime}\right) .
$$

As a result,

$$
\begin{aligned}
& \left|b\left(x, \eta \cup\left\{x^{\prime}\right\}\right)-b(x, \eta)\right| \\
\leq & (1+p) B^{2} G\left(x-x^{\prime}\right) \sum_{y \in \eta} G(x-y) r(y, \eta)+B G^{2}\left(x-x^{\prime}\right) \sup _{s \geq 0}(1+p s) e^{-s} \\
\leq & \text { const } \cdot G\left(x-x^{\prime}\right),
\end{aligned}
$$

where we used Proposition 3.2 with $a$ replaced by $G$.

Hence (2.6) holds, and we get the statement from Theorem 2.6.

Remark 3.4. It is straightforward to check, following the proofs above, that the statements of Proposition 3.2 and Theorem 3.3 remain true if we replace $a(x-y)$ in $(3.1)$ by $a_{1}(x) a_{2}(y) a(x-y)$ with any bounded functions $a_{1}, a_{2}: \mathbb{R}^{\mathrm{d}} \rightarrow \mathbb{R}_{+}$.

\section{Properties of a process with bounded birth rate}

In this Section, we study some general properties of birth-and-death processes which are described in Section 2 and which have globally bounded birth rate $b$. Namely, let the assumptions (2.5)-(2.6) hold and then, by Theorem 2.6, $\eta: \mathbb{R}_{+} \rightarrow \Gamma_{G}$ is the unique solution to (2.4) in the sense of Definition 2.1.

One example of such rate given by (3.1) under Conditions 1-3 was discussed in Theorem 3.3. Consider another examples.

Example 4.1 (Glauber dynamcis in continuum). Consider the rate

$$
b_{z, \phi}(x, \eta)=z \exp \left(-\sum_{y \in \eta \backslash\{x\}} \phi(x-y)\right),
$$

where $z>0$ and $\phi: \mathbb{R}^{\mathrm{d}} \rightarrow \mathbb{R}_{+}$is such that $\phi(x) \leq B G(x), x \in \mathbb{R}^{\mathrm{d}}$ for some $B>0$. Then the mapping (2.3) is the generator of the so-called Glauber dynamics in continuum which was actively studied in recent decades, see e.g. $[5,7,19]$ and references therein. Clearly, since $\phi \geq 0$, the assumption (2.5) is satisfied. Next,

$$
\left|b_{z, \phi}\left(x, \eta \cup\left\{x^{\prime}\right\}\right)-b_{z, \phi}(x, \eta)\right|=b_{z, \phi}(x, \eta)\left(1-e^{-\phi\left(x-x^{\prime}\right)}\right) \leq z \phi\left(x-x^{\prime}\right),
$$

and hence (2.6) holds as well.

An important particular case is when $\phi \equiv 0$, i.e. both death and birth rates are constants. The corresponding process is called a Surgailis process, cf. [28]. 
Example 4.2 (Establishment rate). Consider the rate, cf. (3.1):

$$
b_{a, c, \phi}(x, \eta)=\sum_{y \in \eta} a(x-y)\left(1+\sum_{z \in \eta} c(x-z)\right) \exp \left(-\sum_{z \in \eta} \phi(x-z)\right),
$$

where integrable functions $a, c, \phi: \mathbb{R}^{\mathrm{d}} \rightarrow \mathbb{R}_{+}$are such that, for $x \in \mathbb{R}^{\mathrm{d}}$,

$$
a(x) \leq q \phi(x), \quad c(x) \leq p \phi(x), \quad \phi(x) \leq B G(x),
$$

for some $q, B>0, p \geq 0$. Here if the system is in a state $\eta_{t} \in \Gamma_{G}$ at the time $t \in \mathbb{R}_{+}$, then each $y \in \eta_{t}$ may send an off-spring after exponential random time whose rate is $\langle a\rangle$. The off-spring will be sent according to the probability distribution on $\mathbb{R}^{\mathrm{d}}$ with the (normalized) density $\varkappa^{-1}$ a. However, this off-spring may not survive because of a competition around it. The rate of surviving at $x$ is

$$
\left(1+\sum_{z \in \eta} c(x-z)\right) \exp \left(-\sum_{z \in \eta} \phi(x-z)\right)
$$

The assumptions in (4.2) imply that $b_{a, c, \phi}(x, \eta) \leq g\left(\sum_{z \in \eta} \phi(x-z)\right)$, where $g(s)=q s(1+p s) e^{-s}, s \in \mathbb{R}_{+}$, and hence (2.5) holds. Moreover,

$$
\begin{aligned}
& b_{a, c, \phi}\left(x, \eta \cup\left\{x^{\prime}\right\}\right) \\
= & b_{a, c, \phi}(x, \eta) e^{-\phi\left(x-x^{\prime}\right)} \\
& +a\left(x-x^{\prime}\right) e^{-\phi\left(x-x^{\prime}\right)}\left(1+\sum_{z \in \eta} c(x-z)\right) \exp \left(-\sum_{z \in \eta} \phi(x-z)\right) \\
& +a\left(x-x^{\prime}\right) e^{-\phi\left(x-x^{\prime}\right)} c\left(x-x^{\prime}\right) \exp \left(-\sum_{z \in \eta} \phi(x-z)\right) \\
& +\sum_{y \in \eta} a(x-y) e^{-\phi\left(x-x^{\prime}\right)} c\left(x-x^{\prime}\right) \exp \left(-\sum_{z \in \eta} \phi(x-z)\right) .
\end{aligned}
$$

Then (4.2) implies

$$
\begin{aligned}
& \mid \begin{array}{l}
\left|b_{a, c, \phi}\left(x, \eta \cup\left\{x^{\prime}\right\}\right)-b_{a, c, \phi}(x, \eta)\right| \\
\leq b_{a, c, \phi}(x, \eta)\left|e^{-\phi\left(x-x^{\prime}\right)}-1\right|
\end{array} \quad+2 a\left(x-x^{\prime}\right) \sup _{s \in \mathbb{R}_{+}}(1+p s) e^{-s} \\
& +q c\left(x-x^{\prime}\right) \sup _{s \in \mathbb{R}_{+}} s e^{-s} \leq B_{1} G\left(x-x^{\prime}\right)
\end{aligned}
$$

for some $B_{1}>0$, that yields (2.6).

Proposition 4.3. Let (2.5)-(2.6) hold, and let $\eta_{t}$ be the unique solution to (2.4) in the sense of Definition 2.1. Then there exists a Surgailis process $\xi_{t}$ with the unit death rate and the birth rate $\mathbf{b}$ such that $\xi_{0}=\eta_{0}$ a.s. implies

$$
\eta_{t} \subset \xi_{t} \quad \text { a.s., } t>0 \text {. }
$$


In other words, $\left(\eta_{t}\right)_{t \geq 0}$ is stochastically dominated by the Surgailis process $\left(\xi_{t}\right)_{t \geq 0}$.

Proof. The process $\xi_{t}$ with the unit death rate and the constant birth rate $b(x, \eta) \equiv \mathbf{b}$ evidently satisfies the assumptions (2.5)-(2.6), and hence, by Theorem 2.6, $\xi_{t}$ is the unique solution to

$$
\begin{aligned}
\xi_{t}(B)= & \int_{(0, t] \times B \times \mathbb{R}_{+}^{2}} I_{[0, \mathbf{b}]}(u) I_{[t-s, \infty)}(r) N(d s, d x, d u, d r) \\
& +\int_{B \times \mathbb{R}_{+}} I_{[t, \infty)}(r) \tilde{\eta}_{0}(d x, d r) .
\end{aligned}
$$

Fix some $t>0$. Then, by (2.4), a.s. for an $x \in \eta_{t} \backslash \eta_{0}$ there exist $s, u, r \in(0, t] \times \mathbb{R}_{+}^{2}$ such that

$$
(s, x, u, r) \in N, \quad u \leq b\left(x, \eta_{s-}\right), \quad r>t-s .
$$

Since $b\left(x, \eta_{s-}\right) \leq \mathbf{b},(4.5)$ and (4.6) imply $x \in \xi_{t}$. Similarly, it follows from (2.4) and (4.5) that if $x \in \eta_{t} \cap \eta_{0}$, then also $x \in \xi_{t} \cap \eta_{0}$. Therefore, (4.4) holds.

Corollary 4.4. There exists $C>0$ such that, for a bounded $B \in \mathscr{B}\left(\mathbb{R}^{\mathrm{d}}\right)$,

$$
\mathbb{E}\left\{\left|\eta_{t} \cap B\right|\right\} \leq C \operatorname{vol}(B)
$$

for all $t>0$, provided that (4.7) holds for $t=0$.

Proof. Indeed, by (4.5),

$$
\begin{aligned}
\mathbb{E}\left\{\left|\xi_{t} \cap B\right|\right\} & \leq \operatorname{vol}(B) \mathbf{b} \int_{0}^{t} \int_{t-s}^{\infty} e^{-r} d r d s+\mathbb{E}\left\{\left|\eta_{0} \cap B\right|\right\} \int_{t}^{\infty} e^{-r} d r \\
& =\operatorname{vol}(B) \mathbf{b}\left(1-e^{-t}\right)+\mathbb{E}\left\{\left|\eta_{0} \cap B\right|\right\} e^{-t} \\
& \leq \max \left\{\operatorname{vol}(B) \mathbf{b}, \mathbb{E}\left\{\left|\eta_{0} \cap B\right|\right\}\right\}
\end{aligned}
$$

that implies the statement because of (4.4).

For an integrable function $f: \mathbb{R}^{\mathrm{d}} \rightarrow \mathbb{R}_{+}$, we will use the notation

$$
\langle f\rangle:=\int_{\mathbb{R}^{\mathrm{d}}} f(x) d x<\infty .
$$

Let a non-increasing function $K:(0, \infty) \rightarrow(0, \infty)$ be such that

$$
\begin{gathered}
\lim _{q \rightarrow 0+} K(q)=\infty, \\
\int_{r}^{\infty} K(q) q^{\mathrm{d}-1} d q<\infty, \quad r>0,
\end{gathered}
$$


and let functions $\phi, h: \mathbb{R}^{\mathrm{d}} \rightarrow(0, \infty)$ be such that

$$
\begin{gathered}
C_{1}:=\sup _{x \in \mathbb{R}^{\mathrm{d}}} \int_{\mathbb{R}^{\mathrm{d}}} \phi(y) K(|x-y|) d y<\infty, \\
2 C_{1} \mathbf{b} \phi(x) \leq h(x) \leq G(x), \quad x \in \mathbb{R}^{\mathrm{d}} .
\end{gathered}
$$

We will also assume $\phi$ and $h$ are separated from 0 on each compact subset of $\mathbb{R}^{\mathrm{d}}$.

We define

$$
\begin{aligned}
\psi(x, y) & =\phi(x) \phi(y) K(|x-y|), & & x, y \in \mathbb{R}^{\mathrm{d}}, x \neq y, \\
V(\eta) & =\sum_{\{x, y\} \subset \eta} \psi(x, y) . & & \eta \in \Gamma,
\end{aligned}
$$

and consider the mapping

$$
\Gamma \ni \eta \mapsto W(\eta):=\langle h, \eta\rangle+V(\eta) \in[0, \infty],
$$

where, we recall, $\langle h, \eta\rangle=\sum_{x \in \eta} h(x)$. The assumption (4.9) implies that $\langle h, \eta\rangle<\infty$ for all $\eta \in \Gamma_{G}$. We set

$$
\Theta:=\Theta_{G}:=\left\{\eta \in \Gamma_{G}: V(\eta)<\infty\right\}
$$

We are going to show now that if $\mathbb{E} W\left(\eta_{0}\right)<\infty$ (and hence a.s. $\eta_{0} \in \Theta$ ) then a.s. $\eta_{t} \in \Theta$ for all $t \geq 0$.

Proposition 4.5. Suppose that $\mathbb{E} W\left(\eta_{0}\right)<\infty$. Then $\mathbb{E} W\left(\eta_{t}\right)<\infty, t>0$.

Proof. For each $t \geq 0$, consider the Poisson point process $\Pi_{t}$ (a.k.a. Poisson random measure) on $\mathbb{R}^{\mathrm{d}}$, defined by

$$
\Pi_{t}(B)=\int_{(0, t] \times B \times \mathbb{R}_{+}^{2}} I_{[0, \mathbf{b}]}(u) N(d s, d x, d u, d r), \quad B \in \mathscr{B}\left(\mathbb{R}^{\mathrm{d}}\right) .
$$

By (4.5) and (4.11), a.s.

$$
\begin{aligned}
& \xi_{t} \backslash \eta_{0} \subset \Pi_{t}, \quad t \geq 0, \\
& \Pi_{s} \subset \Pi_{t}, \quad 0 \leq s \leq t .
\end{aligned}
$$

Next, for each $t \geq 0$, the intensity of $\Pi_{t}$ is equal to $\mathbf{b} t$. Then, by (4.9) and the Slivnyak-Mecke theorem,

$$
\mathbb{E}\left\langle h, \Pi_{t}\right\rangle \leq \mathbb{E}\left\langle G, \Pi_{t}\right\rangle=\mathbf{b} t\langle G\rangle<\infty, \quad t \geq 0 .
$$

In particular, a.a. realisations of $\Pi_{t}$ lie in $\Gamma_{G}$ for $t \geq 0$. Similarly, for $t \geq 0$,

$$
\mathbb{E} V\left(\Pi_{t}\right)=(\mathbf{b} t)^{2} \int_{\mathbb{R}^{\mathrm{d}}} \int_{\mathbb{R}^{\mathrm{d}}} \phi(x) \phi(y) K(|x-y|) d x d y \leq C_{1} \mathbf{b}^{2} t^{2}\langle\phi\rangle,
$$


where we used (4.8). As a result,

$$
\mathbb{E} W\left(\Pi_{t}\right)<\infty, \quad t \geq 0,
$$

and hence $\Pi_{t} \in \Theta$ a.s. for $t \geq 0$.

Next, by (4.12) and (4.4), we have

$$
\eta_{t} \subset \eta_{0} \cup \Pi_{t}, \quad t>0,
$$

and hence, by (4.10),

$$
W\left(\eta_{t}\right) \leq W\left(\eta_{0} \cup \Pi_{t}\right)=W\left(\eta_{0}\right)+W\left(\Pi_{t}\right)+\sum_{\substack{x \in \eta_{0} \\ y \in \Pi_{t}}} \psi(x, y) .
$$

Since $\eta_{0}$ and $\Pi_{t}$ are independent, we get, for all $t>0$,

$$
\begin{aligned}
\mathbb{E} W\left(\eta_{t}\right) & \leq \mathbb{E} W\left(\eta_{0}\right)+\mathbb{E} W\left(\Pi_{t}\right)+\mathbb{E} \mathbb{E}\left[\sum_{\substack{x \in \eta_{0} \\
y \in \Pi_{t}}} \psi(x, y) \mid \eta_{0}\right] \\
& =\mathbb{E} W\left(\eta_{0}\right)+\mathbb{E} W\left(\Pi_{t}\right)+\mathbf{b} t \mathbb{E} \sum_{x \in \eta_{0}} \int_{\mathbb{R}^{\mathrm{d}}} \psi(x, y) d y \\
& \leq \mathbb{E} W\left(\eta_{0}\right)+\mathbb{E} W\left(\Pi_{t}\right)+C_{1} \mathbf{b} t \mathbb{E} \sum_{x \in \eta_{0}} \phi(x) \\
& \leq \mathbb{E} W\left(\eta_{0}\right)+\mathbb{E} W\left(\Pi_{t}\right)+\frac{t}{2} \mathbb{E} W\left(\eta_{0}\right)<\infty,
\end{aligned}
$$

where we used again the Slivnyak-Mecke theorem and also (4.8), (4.9), (4.14).

Remark 4.6. We will show below (see Theorem 4.13) a more stronger statement, namely, that $\limsup \mathbb{E} W\left(\eta_{t}\right)$ is finite. To this end, one needs to justify further properties of the process $\left(\eta_{t}\right)_{t \geq 0}$.

Lemma 4.7. For each $\eta \in \Theta$,

$$
|L W(\eta)| \leq \boldsymbol{b}\langle h\rangle+2 W(\eta)<\infty .
$$

Proof. Using the equality

$$
W(\eta \cup\{x\})-W(\eta)=h(x)+\sum_{y \in \eta} \psi(x, y), \quad \eta \in \Theta, x \notin \eta,
$$

and (2.5), (4.8), (4.9), we get, for all $\eta \in \Gamma_{G}$,

$$
\begin{aligned}
& \left|\int_{\mathbb{R}^{\mathrm{d}}} b(x, \eta)(W(\eta \cup\{x\})-W(\eta)) d x\right| \\
\leq & \mathbf{b}\langle h\rangle+\mathbf{b} \sum_{y \in \eta} \phi(y) \int_{\mathbb{R}^{\mathrm{d}}} \phi(x) K(|x-y|) d x \\
\leq & \mathbf{b}\langle h\rangle+\mathbf{b} C_{1} \sum_{y \in \eta} \phi(y) \leq \mathbf{b}\langle h\rangle+\sum_{y \in \eta} h(y) .
\end{aligned}
$$


Next, using the equality

$$
W(\eta \backslash\{x\})-W(\eta)=-h(x)-\sum_{y \in \eta \backslash\{x\}} \psi(x, y), \quad \eta \in \Theta, x \in \eta,
$$

we have, for all $\eta \in \Theta$,

$$
\left|\sum_{x \in \eta}(W(\eta \backslash\{x\})-W(\eta))\right|=\sum_{x \in \eta} h(x)+2 \sum_{\{x, y\} \subset \eta} \psi(x, y) .
$$

Combining (4.17) with (4.19), we get the statement.

Corollary 4.8. Assume that $\mathbb{E} W\left(\eta_{0}\right)<\infty$. Then

$$
\mathbb{E} \int_{0}^{t}\left|L W\left(\eta_{s-}\right)\right| d s<\infty, \quad t \geq 0
$$

Proof. By Proposition 4.5, a.s. $\eta_{t} \in \Theta$ for all $t>0$. Then, by Lemma 4.7, to prove (4.20), it is enough to show that $\mathbb{E} \int_{0}^{t} W\left(\eta_{s-}\right) d s$ is finite. The latter expression is estimated, because of (4.15), by

$$
\left(t+\frac{t^{2}}{4}\right) \mathbb{E} W\left(\eta_{0}\right)+\mathbb{E} \int_{0}^{t} W\left(\Pi_{s-}\right) d s \leq\left(t+\frac{t^{2}}{4}\right) \mathbb{E} W\left(\eta_{0}\right)+t \mathbb{E} W\left(\Pi_{t}\right)<\infty,
$$

where we used (4.13) and (4.14).

Define $N_{b}$ as the projection of $N$ on first, second, and fourth coordinates. Then, in particular,

$$
N_{b}(\{(s, x, u)\})=1 \Longleftrightarrow \exists r>0: N(\{(s, x, u, r)\})=1 .
$$

Since $\int_{\mathbb{R}_{+}} e^{-r} d r=1, N_{b}$ is a Poisson point process on $\mathbb{R}_{+} \times \mathbb{R}^{\mathrm{d}} \times \mathbb{R}_{+}$with mean measure $d s \times d x \times d u$.

Let $\mathscr{B}_{b}\left(\mathbb{R}^{\mathrm{d}}\right)$ denote the set of all bounded Borel subsets of $\mathbb{R}^{\mathrm{d}}$. Define, for $t \geq 0$,

$$
\begin{aligned}
\mathscr{F}_{t}^{o}:=\sigma\left(N_{b}(A \times U), \eta_{s}(B) \mid\right. & A \in \mathscr{B}((0, t]), \\
U & \left.\in \mathscr{B}\left(\mathbb{R}^{\mathrm{d}} \times \mathbb{R}_{+}\right), s \in[0, t] \cap \mathbb{Q}, B \in \mathscr{B}_{b}\left(\mathbb{R}^{\mathrm{d}}\right)\right),
\end{aligned}
$$

and let $\mathscr{F}_{t}$ be the completion of $\mathscr{F}_{t}^{o}$ under $\mathbb{P}$. Then $N_{b}$ is compatible with $\mathscr{F}_{t}$.

For $B \in \mathscr{B}_{b}\left(\mathbb{R}^{\mathrm{d}}\right)$, define $D_{t}(B)$ as the number of deaths that occured in $B$ up to time $t>0$, i.e.

$$
D_{t}(B):=\left|\left\{s \in(0, t]||\left(\eta_{s-} \backslash \eta_{s}\right) \cap B \mid=1\right\}\right| .
$$


Then, for a fixed $B \in \mathscr{B}_{b}\left(\mathbb{R}^{\mathrm{d}}\right),\left(D_{t}(B)\right)_{t \geq 0}$ is an a.s. finite increasing $\left\{\mathscr{F}_{t}, t \geq\right.$ $0\}$-adapted process, in particular, it is a sub-martingale. Moreover,

$$
D_{t}(B) \leq \eta_{0}(B)+\Pi_{t}(B) .
$$

In the sequel, we will need the assumption that $\mathbb{E} \eta_{0}(B)<\infty$ for all $B \in \mathscr{B}_{b}\left(\mathbb{R}^{\mathrm{d}}\right)$. Note that if $\mathbb{E} W\left(\eta_{0}\right)<\infty$ then the former inequality always holds as we assumed that $h$ is separated from 0 on each compact set, and hence $\mathbb{E} \eta_{0}(B) \leq \alpha \mathbb{E}\left\langle h, \eta_{0}\right\rangle \leq \alpha \mathbb{E} W\left(\eta_{0}\right)<\infty$ for some $\alpha=\alpha(B)>0$.

Under this assumption, (4.21) implies that, for a fixed $B \in \mathscr{B}_{b}\left(\mathbb{R}^{\mathrm{d}}\right)$, the process $\left(D_{t}(B)\right)_{t \geq 0}$ is uniformly integrable on finite time intervals. Therefore by Doob-Meyer decomposition theorem there exists a unique predictable increasing process $\left(A_{t}(B)\right)_{t \geq 0}$ such that $D_{t}(B)-A_{t}(B)$ is a martingale.

Lemma 4.9. Let $B \in \mathscr{B}_{b}\left(\mathbb{R}^{\mathrm{d}}\right)$ and $\mathbb{E} \eta_{0}(B)<\infty$. Then

$$
A_{t}(B)=\int_{0}^{t} \eta_{s-}(B) d s, \quad t \geq 0 .
$$

Proof. By (2.4), $D_{t}(B)=S_{1}(t)+S_{2}(t)$, where

$$
\begin{aligned}
& S_{1}(t):=N\left(\left\{(s, x, u, r) \mid s \in(0, t], u \leq b\left(x, \eta_{s-}\right), x \in B, r \leq t-s\right\}\right), \\
& S_{2}(t):=\tilde{\eta}_{0}(\{(x, r) \mid x \in B, r \leq t-s\}) .
\end{aligned}
$$

By (2.4), a.s.

$$
x \in \eta_{s} \backslash \eta_{s-} \Longleftrightarrow \exists u \in\left[0, b\left(x, \eta_{s-}\right)\right], r \geq 0:(s, x, u, r) \in N .
$$

Note that since a.s.

$$
\sup _{x \in \mathbb{R}^{\mathrm{d}}} N(\{(s, x, u, r) \mid s, u, r \geq 0\})=1,
$$

and

$$
N\left(\left\{(s, x, u, r) \mid s, u, r \geq 0, x \in \eta_{0}\right\}\right)=0,
$$

we can conclude that a.s. there does not exist $x$ and $\left(s_{1}, u_{1}, r_{1}\right) \neq\left(s_{2}, u_{2}, r_{2}\right)$ such that $\left(s_{i}, x, u_{i}, r_{i}\right) \in N, i=1,2$. Hence by (4.23)-(4.25) a.s.

$$
\begin{aligned}
& N\left(\left\{(s, x, u, r) \mid s \in(0, t], x \in \eta_{s}, u>b\left(x, \eta_{s^{-}}\right), x \in B\right\}\right) \\
\leq & N\left(\left\{(s, x, u, r) \mid s \in(0, t], x \in \eta_{0}, u>b\left(x, \eta_{s^{-}}\right), x \in B\right\}\right) \\
& +N\left(\left\{(s, x, u, r) \mid s \in(0, t], x \in \eta_{s} \backslash \eta_{0}, u>b\left(x, \eta_{s-}\right), x \in B\right\}\right)=0 .
\end{aligned}
$$


Therefore, a.s.

$$
S_{1}(t)=N\left(\left\{(s, x, u, r) \mid s \in(0, t], x \in \eta_{s}, x \in B, r \leq t-s\right\}\right) .
$$

Note also that both $S_{1}$ and $S_{2}$ are, evidently, increasing processes.

We are going to show firstly that

$$
S_{1}(t)-\int_{0}^{t}\left|\left(\eta_{s-} \backslash \eta_{0}\right) \cap B\right| d s
$$

is a martingale. To this end we write

$$
\begin{aligned}
& S_{1}(t+\Delta t)-S_{1}(t) \\
= & N\left(\left\{(s, x, u, r) \mid s \in(0, t], x \in\left(\eta_{s} \backslash \eta_{0}\right), x \in B, t-s<r \leq t+\Delta t-s\right\}\right) \\
& +N\left(\left\{(s, x, u, r) \mid s \in(t, t+\Delta t], x \in\left(\eta_{s} \backslash \eta_{0}\right), x \in B, r \leq t+\Delta t-s\right\}\right) \\
= & : S_{3}(t, t+\Delta t)+S_{4}(t, t+\Delta t) .
\end{aligned}
$$

Note that $S_{3}(t, t+\Delta t)$ is the number of particles born during $(0, t]$ dying during $(t, t+\Delta t]$, and $S_{4}(t, t+\Delta t)$ is the number of particles who both are being born and die during $(t, t+\Delta t$. Since the lifespan of every particle is a unit exponential, for every $T>0$ a.s.

$$
\lim _{\Delta t \rightarrow 0} \sup _{t \in[0, T]} S_{4}(t, t+\Delta t)=0
$$

and also

$$
\mathbb{P}\left\{S_{4}(t, t+\Delta t)>0\right\}=o(\Delta t), \quad t>0 .
$$

For $x \in \eta_{t} \backslash \eta_{0}$, the 'residual clock times' (see also [14]) $r-(t-s)$ are independent of $\mathscr{F} t$ by the properties of a Poisson point process, and hence the residual clock times have the same unit exponential distribution. Therefore, conditionally on $\mathscr{F} t, S_{3}(t, t+\Delta t)$ has the binominal distribution with parameters $\left|\left(\eta_{t} \backslash \eta_{0}\right) \cap B\right|$ and $1-e^{-\Delta t}$. Consequently,

$$
\begin{aligned}
& \mathbb{P}\left[S_{1}(t+\Delta t)-S_{1}(t)=1 \mid \mathscr{F}_{t}\right]=\left|\left(\eta_{t} \backslash \eta_{0}\right) \cap B\right| \Delta t+o(\Delta t), \\
& \mathbb{P}\left[S_{1}(t+\Delta t)-S_{1}(t)=0 \mid \mathscr{F}_{t}\right]=1-\left|\left(\eta_{t} \backslash \eta_{0}\right) \cap B\right| \Delta t+o(\Delta t), \\
& \mathbb{P}\left[S_{1}(t+\Delta t)-S_{1}(t)>1 \mid \mathscr{F}_{t}\right]=o(\Delta t),
\end{aligned}
$$

Thus, $\left(S_{1}(t)\right)_{t \geq 0}$ is a pure jump type process with unit jumps, and it follows from (4.27)-(4.29) that the rates of jumps at time $t$ are given by $\left|\left(\eta_{t} \backslash \eta_{0}\right) \cap B\right|$. Hence the process in (4.26) is indeed a martingale.

Similarly one can show that $S_{2}(t)-\int_{0}^{t}\left|\eta_{s-} \cap \eta_{0} \cap B\right| d s$ is a martingale. Therefore,

$$
D_{t}(B)-\int_{0}^{t} \eta_{s}(B) d s=D_{t}(B)-\int_{0}^{t} \eta_{s-}(B) d s
$$


is also a martingale.

Remark 4.10. It follows from Lemma 4.9 that the point process $D$ on $\mathbb{R}_{+} \times \mathbb{R}^{\mathrm{d}}$ defined by $(t, x) \in D \Leftrightarrow x \in \eta_{t-} \backslash \eta_{t}$ can be viewed as a point process with the predictable compensator given by (4.22).

Proposition 4.11. Suppose that $\mathbb{E} W\left(\eta_{0}\right)<\infty$. The process

$$
M_{t}:=W\left(\eta_{t}\right)-\int_{0}^{t} L W\left(\eta_{s}\right) d s
$$

is an $\left(\mathscr{F}_{t}\right)$-martingale.

Proof. Let $B \in \mathscr{B}_{b}\left(\mathbb{R}^{\mathrm{d}}\right)$. Then

$$
\begin{aligned}
& W\left(\eta_{t} \cap B\right)-W\left(\eta_{0}\right) \\
= & \int_{(0, t] \times B \times \mathbb{R}_{+}} I_{\left[0, b\left(x, \eta_{s-}\right)\right]}(u)\left\{W\left(\eta_{s-} \cup\{x\}\right)-W\left(\eta_{s-}\right)\right\} N_{b}(d s, d x, d u) \\
& +\int_{(0, t] \times B}\left\{W\left(\eta_{s-} \backslash\{x\}\right)-W\left(\eta_{s-}\right)\right\} D(d s, d x) \\
= & \int_{(0, t] \times B \times \mathbb{R}_{+}} I_{\left[0, b\left(x, \eta_{s-}\right)\right]}(u)\left\{W\left(\eta_{s-} \cup\{x\}\right)-W\left(\eta_{s-}\right)\right\}\left(N_{b}(d s, d x, d u)-d s d x d u\right) \\
& +\int_{(0, t] \times B} b\left(x, \eta_{s-}\right)\left\{W\left(\eta_{s-} \cup\{x\}\right)-W\left(\eta_{s-}\right)\right\} d s d x \\
& +\int_{(0, t] \times B}\left\{W\left(\eta_{s-} \backslash\{x\}\right)-W\left(\eta_{s-}\right)\right\}\left(D(d s, d x)-d s \eta_{s-}(d x)\right) \\
& +\int_{(0, t] \times B}\left\{W\left(\eta_{s-} \backslash\{x\}\right)-W\left(\eta_{s-}\right)\right\} d s \eta_{s-}(d x) .
\end{aligned}
$$

By Lemma 4.9, the integrals with respect to $N_{b}(d s, d x, d u)-d s d x d u$ and $D(d s, d x)-d s \eta_{s-}(d x)$ are martingales as integrals with respect to the difference between a point process and its compensator, see e.g. [15, (3.8), Chapter 2]. Therefore,

$$
\begin{aligned}
W\left(\eta_{t} \cap B\right) & -\int_{(0, t] \times B} b\left(x, \eta_{s-}\right)\left\{W\left(\eta_{s-} \cup\{x\}\right)-W\left(\eta_{s-}\right)\right\} d s d x \\
& -\int_{(0, t]} d s \sum_{x \in \eta_{s-} \cap B}\left\{W\left(\eta_{s-} \backslash\{x\}\right)-W\left(\eta_{s-}\right)\right\}
\end{aligned}
$$


is indeed an $\left(\mathscr{F}_{t}\right)$-martingale. The statement of the Lemma follows then from the dominated convergence theorem by using Proposition 4.5 and Corollary 4.8 .

The following lemma shows that the function $W$ is of the Foster-Lyapunov type.

Lemma 4.12. For all $\eta \in \Theta$,

$$
L W(\eta) \leq \boldsymbol{b}\langle h\rangle-\frac{1}{2} W(\eta) .
$$

Proof. By (2.3), (4.16), (4.18), for each $\eta \in \Theta$, we have

$$
\begin{aligned}
L W(\eta)= & -\sum_{z \in \eta} h(z)-2 \sum_{\{x, y\} \subset \eta} \psi(x, y) \\
& +\int_{\mathbb{R}^{\mathrm{d}}} b(x, \eta) h(x) d x+\sum_{y \in \eta_{\mathbb{R}^{\mathrm{d}}}} \int b(x, \eta) \psi(x, y) d x .
\end{aligned}
$$

Then, by (2.5), (4.8), (4.9),

$$
\begin{aligned}
L W(\eta) & \leq-\sum_{z \in \eta} h(z)-2 \sum_{\{x, y\} \subset \eta} \psi(x, y)+\mathbf{b}\langle h\rangle+C_{1} \mathbf{b} \sum_{y \in \eta} \phi(y) \\
& \leq \mathbf{b}\langle h\rangle-\frac{1}{2} \sum_{z \in \eta} h(z)-2 \sum_{\{x, y\} \subset \eta} \psi(x, y),
\end{aligned}
$$

that yields (4.31).

Theorem 4.13. Suppose that $\mathbb{E} W\left(\eta_{0}\right)<\infty$. Then

$$
\limsup _{t \rightarrow \infty} \mathbb{E} W\left(\eta_{t}\right) \leq 2 \boldsymbol{b}\langle h\rangle .
$$

Proof. By Proposition 4.5, $w(t):=\mathbb{E} W\left(\eta_{t}\right)<\infty$ for $t>0$. By Proposition $4.11,(4.30)$ defines a martingale. Taking the expectation in (4.30), we get

$$
w(t)=\mathbb{E} \int_{0}^{t} L W\left(\eta_{s}\right) d s+w(0) .
$$

By (4.33) and Corollary 4.8, $w$ is differentiable. Taking the derivative, we obtain

$$
w^{\prime}(t)=\mathbb{E} L W\left(\eta_{t}\right) \leq \mathbf{b}\langle h\rangle-\frac{1}{2} w(t),
$$

by (4.31). By the comparison principle,

$$
w(t) \leq e^{-\frac{1}{2} t} w(0)+\mathbf{b}\langle h\rangle \int_{0}^{t} e^{-\frac{1}{2}(t-s)} d s \leq e^{-\frac{1}{2} t} w(0)+2 \mathbf{b}\langle h\rangle,
$$

that yields (4.32). 
Now we are going to apply the techniques similar to the considered in [24]. Let $\delta>0$ be a small number. For $K>0$, let $\tau_{K}$ be the return times to the set $\{\zeta \in \Theta: W(\zeta)<K\}$, namely,

$$
\tau_{K}=\inf \left\{t>\delta \mid W\left(\eta_{t}\right)<K\right\}
$$

Proposition 4.14. Suppose that $\mathbb{E} W\left(\eta_{0}\right)<\infty$. Then, for each $K>\boldsymbol{b}\langle h\rangle$,

$$
\mathbb{E} \tau_{K} \leq \frac{\mathbb{E} W\left(\eta_{0}\right)}{K-\boldsymbol{b}\langle h\rangle}
$$

Proof. Denote $\kappa=K-\mathbf{b}\langle h\rangle>0$. By (4.31) and (4.34), we have a.s. on $\left\{t<\tau_{K}\right\}$

$$
L W\left(\eta_{t}\right) \leq \mathbf{b}\langle h\rangle-K=-\kappa .
$$

Next, by (4.30),

$$
\mathbb{E} W\left(\eta_{\tau_{K} \wedge t}\right)-\mathbb{E} \int_{0}^{\tau_{K} \wedge t} L W\left(\eta_{s}\right) d s=\mathbb{E} W\left(\eta_{0}\right),
$$

hence, by (4.35),

$$
\mathbb{E} W\left(\eta_{0}\right) \geq \mathbb{E} W\left(\eta_{\tau_{K} \wedge t}\right)+\kappa \mathbb{E} \tau_{K} \wedge t
$$

and, therefore,

$$
\kappa \mathbb{E} \tau_{K} \wedge t \leq \mathbb{E} W\left(\eta_{0}\right)-\mathbb{E} W\left(\eta_{\tau_{K} \wedge t}\right) \leq \mathbb{E} W\left(\eta_{0}\right) .
$$

Taking $t \rightarrow \infty$, we get the desired result.

The next proposition shows the existence of an exponential moment of $\tau_{K}$ for sufficiently large $K$.

Proposition 4.15. Assume that $\mathbb{E} W\left(\eta_{0}\right)<\infty$. Then, for all $\theta \in\left(0, \frac{1}{2}\right)$, there exists $K_{\theta}>0$ such that, for $K>K_{\theta}$,

$$
\mathbb{E} e^{\theta \tau_{K}}<\infty
$$

Proof. Fix any $\theta \in\left(0, \frac{1}{2}\right)$, and define

$$
\Phi(t, x):=e^{\theta t}(x+1) \geq 1, \quad t, x \in \mathbb{R}_{+} .
$$

We are now going to show that

$$
\Phi\left(t, W\left(\eta_{t}\right)\right)-\int_{0}^{t} \mathbb{L} \Phi\left(s, W\left(\eta_{s}\right)\right) d s
$$


is a local martingale, where

$$
\mathbb{L} \Phi\left(s, W\left(\eta_{s}\right)\right):=\theta \Phi\left(s, W\left(\eta_{s}\right)\right)+e^{\theta s} L W\left(\eta_{s}\right) .
$$

By [4, Proposition 3.2, Chapter 2] and since $e^{\theta t}$ is locally of bounded variation, the process

$$
\begin{aligned}
\widetilde{M}_{t}:= & e^{\theta t}\left[W\left(\eta_{t}\right)+1-\int_{0}^{t} L W\left(\eta_{s}\right) d s\right] \\
& -\int_{0}^{t}\left[W\left(\eta_{s}\right)+1-\int_{0}^{s} L W\left(\eta_{u}\right) d u\right] \theta e^{\theta s} d s
\end{aligned}
$$

is a local martingale. Now,

$$
\begin{gathered}
e^{\theta t} \int_{0}^{t} L W\left(\eta_{s}\right) d s-\int_{0}^{t} \int_{0}^{s} L W\left(\eta_{u}\right) d u \theta e^{\theta s} d s \\
=e^{\theta t} \int_{0}^{t} L W\left(\eta_{s}\right) d s-\int_{0}^{t} L W\left(\eta_{u}\right) d u \int_{u}^{t} \theta e^{\theta s} d s \\
=e^{\theta t} \int_{0}^{t} L W\left(\eta_{s}\right) d s-\int_{0}^{t} L W\left(\eta_{u}\right)\left(e^{\theta t}-e^{\theta u}\right) d u=\int_{0}^{t} e^{\theta u} L W\left(\eta_{u}\right) d u,
\end{gathered}
$$

hence from (4.37)

$$
\begin{aligned}
\widetilde{M}_{t} & =e^{\theta t}\left[W\left(\eta_{t}\right)+1\right]-\int_{0}^{t}\left[W\left(\eta_{s}\right)+1\right] \theta e^{\theta s} d s-\int_{0}^{t} e^{\theta s} L W\left(\eta_{s}\right) d s \\
& =\Phi\left(t, W\left(\eta_{t}\right)\right)-\int_{0}^{t} \theta \Phi\left(t, W\left(\eta_{s}\right)\right) d s-\int_{0}^{t} e^{\theta s} L W\left(\eta_{s}\right) d s,
\end{aligned}
$$

and we see that the process in (4.36) is indeed a local martingale.

By Lemma 4.12,

$$
\mathbb{L} \Phi\left(s, W\left(\eta_{s}\right)\right) \leq e^{\theta s}\left[\theta W\left(\eta_{s}\right)+\theta+\mathbf{b}\langle h\rangle-\frac{1}{2} W\left(\eta_{s}\right)\right] .
$$

Take a localizing sequence of stopping times $\left\{\sigma_{n}\right\}$ or the martingale (4.36), 
$\sigma_{n} \nearrow \infty, n \rightarrow \infty$, a.s. Then

$$
\begin{aligned}
& \mathbb{E} e^{\theta \tau_{K} \wedge t \wedge \sigma_{n}} \\
\leq & \mathbb{E} e^{\theta \tau_{K} \wedge t \wedge \sigma_{n}}\left[W\left(\eta_{\theta \tau_{K} \wedge t \wedge \sigma_{n}}\right)+1\right] \\
= & \mathbb{E} \Phi\left(\tau_{K} \wedge t \wedge \sigma_{n}, W\left(\eta_{\tau_{K} \wedge t \wedge \sigma_{n}}\right)\right) \\
= & \mathbb{E} \Phi\left(0, W\left(\eta_{0}\right)\right)+\mathbb{E} \int_{0}^{\tau_{K} \wedge t \wedge \sigma_{n}} \mathbb{L} \Phi\left(s, W\left(\eta_{s}\right)\right) d s \\
\leq & \mathbb{E} W\left(\eta_{0}\right)+\mathbb{E} \int_{0}^{\tau_{K} \wedge t \wedge \sigma_{n}} e^{\theta s}\left[\theta W\left(\eta_{s}\right)+\theta+\mathbf{b}\langle h\rangle-\frac{1}{2} W\left(\eta_{s}\right)\right] d s \\
\leq & \mathbb{E} W\left(\eta_{0}\right)+\mathbb{E} \int_{0}^{\tau_{K} \wedge t \wedge \sigma_{n}} e^{\theta s}\left(\left(\theta-\frac{1}{2}\right) K+\theta+\mathbf{b}\langle h\rangle\right) d s .
\end{aligned}
$$

Therefore, for $K>(\theta+\mathbf{b}\langle h\rangle)\left(\frac{1}{2}-\theta\right)^{-1}$ we get

$$
\mathbb{E} e^{\theta \tau_{K} \wedge t \wedge \sigma_{n}} \leq \mathbb{E} W\left(\eta_{0}\right) .
$$

Taking here $n \rightarrow \infty$ and then $t \rightarrow \infty$ concludes the proof.

\section{Uniqueness of the degenerate invariant distribu- tion for sublinear birth rate}

In this Section, we study some general properties of birth-and-death processes which are described in Section 2 and which have sublinear birth rate $b$, namely,

$$
b(x, \eta) \leq \sum_{y \in \eta} g(x-y), \quad \eta \in \Gamma_{G}, x \notin \eta,
$$

for some $g: \mathbb{R}^{\mathrm{d}} \rightarrow(0, \infty)$, such that $g(x) \leq B G(x), x \in \mathbb{R}^{\mathrm{d}}$, with some $B>0$. We will assume that $\left(\eta_{t}\right)_{t \geq 0}$ is the unique solution to $(2.4)$ in the sense of Definition 2.1.

We are going to find sufficient conditions for $g$ such that

$$
\langle g\rangle<1
$$

would imply that the Dirac measure concentrated at $\varnothing$ is the only invariant distribution for $\eta_{t}$ on $\Gamma_{G}$.

Note that, (5.1) implies $b(x, \varnothing)=0$ and hence the empty configuration is a trap. Therefore, the Dirac measure concentrated at $\varnothing$ is indeed an invariant distribution for $\left(\eta_{t}\right)$, so one need to show the uniqueness only. 
Again, our first example is the rate given by (3.1) under Conditions 1-3. Then Condition 3 implies (5.1) with

$$
g(x)=r_{p} a(x), \quad x \in \mathbb{R}^{\mathrm{d}},
$$

where

$$
r_{p}:=\sup _{s \in \mathbb{R}_{+}}(1+p s) e^{-s}= \begin{cases}1, & 0 \leq p \leq 1, \\ p e^{\frac{1}{p}-1}, & p>1,\end{cases}
$$

i.e. (5.2) takes the form

$$
r_{p}\langle a\rangle<1 .
$$

Another example is the establishment birth rate (4.1). The condition $c(x) \leq p \phi(x), x \in \mathbb{R}^{\mathrm{d}}$, with $p \geq 0$, cf. (4.2), implies that the surviving rate (4.3) is bounded by (5.4), and hence (5.1) also holds with $g$ given by (5.3).

Finally, an evident example is $b(x, \eta)=\sum_{y \in \eta} g(x-y)$. The corresponding process is then a special form of a spatial branching (nonlocal) process (when each particle $y$ may die and produce zero or two off-springs: one of them is at the same position $y$ and another is distributed according to the kernel $g$ ), also known as a contact process in the continuum, see [2,21]. This rate is not bounded and does not satisfy the assumption (2.5) of Theorem 2.6, however, it is straightforward to check that it satisfies the condtions of [14, Theorem 2.13, Lemma 3.14] and hence the statement of Theorem 2.6 holds true for it. Note that then the assumption (5.2) describes the socalled sub-critical regime when the process 'dies out', see e.g. [2, Section 3]. For another models on the configuration space where the uniqueness of the invariant measures was sorted out, see e.g. [10,11].

Theorem 5.1. Let (5.1) hold with

$$
g(x)=b_{g}(|x|) \leq C(1+|x|)^{-\mathrm{d}-2 \varepsilon}, \quad x \in \mathbb{R}^{\mathrm{d}},
$$

for some $C>0$, where $b_{g}: \mathbb{R}_{+} \rightarrow(0, \infty)$ is a continuously decreasing to 0 function. Suppose also that (5.2) holds. Then the Dirac measure concentrated at $\varnothing$ is the only invariant distribution for $\eta_{t}$ on $\Gamma_{G}$.

Proof. Recall, that we have to show that the uniqueness of the invariant distribution only. Note also that (5.5) yields $g(x) \leq C G(x), x \in \mathbb{R}^{\mathrm{d}}$.

Let $\sigma_{d}$ denote the surface area of a unit sphere in $\mathbb{R}^{\mathrm{d}}$; then, by (5.5) and (5.2),

$$
\langle g\rangle=\sigma_{d} \int_{0}^{\infty} b_{g}(s) s^{\mathrm{d}-1} d s<1 .
$$

Choose $R>0$, such that $C \leq(1+R)^{\frac{1}{2} \varepsilon}$ and also

$$
\sigma_{d} \int_{R}^{\infty}(1+s)^{-\mathrm{d}-\frac{3}{2} \varepsilon} s^{\mathrm{d}-1} d s<1-\langle g\rangle .
$$


Then, for $|x| \geq R$,

$$
b_{g}(|x|) \leq \frac{C}{(1+|x|)^{\mathrm{d}+2 \varepsilon}} \leq \frac{(1+R)^{\frac{1}{2} \varepsilon}}{(1+|x|)^{\frac{1}{2} \varepsilon}} \frac{1}{(1+|x|)^{\mathrm{d}+\frac{3}{2} \varepsilon}} \leq \frac{1}{(1+|x|)^{\mathrm{d}+\frac{3}{2} \varepsilon}} .
$$

Let $R_{1} \geq R$ satisfy

$$
\left(1+R_{1}\right)^{-\mathrm{d}-\frac{3}{2} \varepsilon}=b_{g}(R)
$$

Set

$$
c_{g}(x)= \begin{cases}b_{g}(|x|), & |x| \leq R, \\ b_{g}(R), & R \leq|x| \leq R_{1}, \\ (1+|x|)^{-\mathrm{d}-\frac{3}{2} \varepsilon,} & |x| \geq R_{1} .\end{cases}
$$

As a result, $c_{g}: \mathbb{R}^{\mathrm{d}} \rightarrow(0, \infty)$ is a radially symmetric continuous bounded integrable function, such that

$$
g(x)=b_{g}(|x|) \leq c_{g}(x) \leq \begin{cases}b_{g}(|x|), & |x| \leq R, \\ (1+|x|)^{-\mathrm{d}-\frac{3}{2} \varepsilon}, & |x| \geq R,\end{cases}
$$

and therefore, by (5.6)

$$
\begin{aligned}
\left\langle c_{g}\right\rangle & \leq \int_{\{|x| \leq R\}} b_{g}(|x|) d x+\int_{\{|x| \geq R\}} \frac{1}{(1+|x|)^{\mathrm{d}+\frac{3}{2} \varepsilon}} d x \\
& \leq\langle g\rangle+\sigma_{d} \int_{R}^{\infty}(1+s)^{-\mathrm{d}-\frac{3}{2} \varepsilon} s^{\mathrm{d}-1} d s<1 .
\end{aligned}
$$

Let $*$ denote the convolution of functions over $\mathbb{R}^{\mathrm{d}}$. Set, for each $n \in \mathbb{N}$, $c_{g}^{* n}(x)=\left(c_{g} * \ldots * c_{g}\right)(x)(n-1$ times). It is straightforward to check that the normalised function $\left\langle c_{g}\right\rangle^{-1} c_{g}$ satisfies the assumptions of [9, Theorem 4.1]. Then there exists $\alpha_{0} \in(0,1)$ such that, for any $\delta \in(0,1)$ and $\alpha \in\left(\alpha_{0}, 1\right)$, there exist $B_{1}=B_{1}(\delta, \alpha)>0$ and $\lambda=\lambda(\delta, \alpha) \in(0,1)$, such that, for all $n \in \mathbb{N}$,

$$
c_{g}^{* n}(x) \leq B_{1}\left\langle c_{g}\right\rangle^{n}(1+\delta)^{n} \min \left\{\lambda, c_{g}(|x|)^{\alpha}\right\}, \quad x \in \mathbb{R}^{\mathrm{d}} .
$$

By (5.8), one can fix a $\delta \in(0,1)$ such that

$$
\left\langle c_{g}\right\rangle(1+\delta)<1 .
$$

Choose also some $\alpha \in\left(\alpha_{0}, 1\right)$ such that

$$
\alpha\left(\mathrm{d}+\frac{3}{2} \varepsilon\right)>\mathrm{d}+\varepsilon
$$

Then, by (5.7),

$$
c_{g}(|x|)^{\alpha} \leq K G(x), \quad x \in \mathbb{R}^{\mathrm{d}}
$$

for some $K>0$. 
By (5.9), (5.10), (5.11), the series

$$
f(x):=c_{g}(x)+\sum_{n=2}^{\infty} c_{g}^{* n}(x), \quad x \in \mathbb{R}^{\mathrm{d}}
$$

converges uniformly on each compact of $\mathbb{R}^{\mathrm{d}}$ and its sum $f$ is a continuous function such that $f(x) \leq K_{1} G(x), x \in \mathbb{R}^{\mathrm{d}}$, for some $K_{1}>0$. In particular, of course, $f$ is integrable and bounded on $\mathbb{R}^{\mathrm{d}}$. Moreover, $f$ satisfies the equality

$$
f(x)=c_{g}(x)+\left(c_{g} * f\right)(x), \quad x \in \mathbb{R}^{\mathrm{d}} .
$$

Set

$$
F(\eta):=\langle f, \eta\rangle, \quad \eta \in \Gamma_{G} .
$$

Recall that $L$ is given by (2.3), then, for $\eta \in \Gamma_{G}$, (5.1), (5.7), and (5.12) imply that

$$
\begin{aligned}
L F(\eta) & \leq-\langle f, \eta\rangle+\sum_{y \in \eta} \int_{\mathbb{R}^{\mathrm{d}}} g(x-y) f(x) d x \\
& \leq\left\langle c_{g} * f, \eta\right\rangle-\langle f, \eta\rangle=-\left\langle c_{g}, \eta\right\rangle .
\end{aligned}
$$

Assume firstly that $\mathbb{E} F\left(\eta_{0}\right)<\infty$. Similarly to the proof of Proposition 4.11 , one can show that $F\left(\eta_{t}\right)-\int_{0}^{t} L F\left(\eta_{s-}\right) d s$ is a martingale. Then (5.13) implies that $F\left(\eta_{t}\right)+\int_{0}^{t}\left\langle c_{g}, \eta_{s-}\right\rangle d s$ is a non-negative supermartingale. Hence, by Doob's martingale convergence theorem, a.s.

$$
\int_{0}^{\infty}\left\langle c_{g}, \eta_{s-}\right\rangle d s<\infty
$$

Furthermore,

$$
\mathbb{E} \int_{0}^{\infty}\left\langle c_{g}, \eta_{s-}\right\rangle d s=\lim _{t \rightarrow \infty} \mathbb{E} \int_{0}^{t}\left\langle c_{g}, \eta_{s-}\right\rangle d s \leq \mathbb{E} F\left(\eta_{0}\right)<\infty .
$$

Consequently, if $\mu$ is an invariant distribution for $\left(\eta_{t}\right)_{t \geq 0}$ satisfying

$$
\int_{\Gamma_{G}}\langle f, \eta\rangle \mu(d \eta)<\infty
$$

then $\mu$ is the Dirac measure concentrated at the empty configuration since otherwise $\mathbb{E} \int_{0}^{\infty}\left\langle c_{g}, \eta_{s-}\right\rangle d s=\infty$ if $\eta_{0}$ is distributed according to $\mu$, contradicting to (5.14). 
We have shown that, apart from the delta measure at $\varnothing$, there is no invariant distribution $\mu$ with $\int_{\Gamma_{G}}\langle f, \eta\rangle \mu(d \eta)<\infty$. Assume that there exists an invariant distribution $\mu$ with $\int_{\Gamma_{G}}\langle f, \eta\rangle \mu(d \eta)=\infty$.

Let $\eta_{0}$ be distributed according to $\mu$. Define

$$
\eta_{0}^{M}= \begin{cases}\eta_{0}, & \text { if }\left\langle f, \eta_{0}\right\rangle \leq M \\ \varnothing, & \text { otherwise }\end{cases}
$$

Consider now the process $\left(\eta_{t}^{M}\right)_{t \geq 0}$ started from $\eta_{0}^{M}$. By the uniquness of solutions to (2.4), on the event $\left\{\eta_{0}^{M}=\eta_{0}\right\}$ we have a.s. $\left\{\eta_{t}^{M}=\eta_{t}, t \geq 0\right\}$. The arguments leading to (5.14) can be applied to $\left(\eta_{t}^{M}\right)_{t \geq 0}$. As a result,

$$
\mathbb{E} \int_{0}^{\infty}\left\langle c_{g}, \eta_{s-}^{M}\right\rangle d s=\lim _{t \rightarrow \infty} \mathbb{E} \int_{0}^{t}\left\langle c_{g}, \eta_{s-}^{M}\right\rangle d s \leq \mathbb{E} F\left(\eta_{0}^{M}\right) \leq M .
$$

From (5.15) it follows that, for every $\varepsilon>0$,

$$
\begin{aligned}
& \inf _{t \geq 0} \mathbb{P}\left\{\left\langle c_{g}, \eta_{t-}^{M}\right\rangle>\varepsilon\right\} \leq \liminf _{t \rightarrow \infty} \frac{\mathbb{E}\left\langle c_{g}, \eta_{t-}^{M}\right\rangle}{\varepsilon} \\
& \leq \liminf _{t \rightarrow \infty} \frac{\mathbb{E} F\left(\eta_{0}^{M}\right)}{t \varepsilon} \leq \liminf _{t \rightarrow \infty} \frac{\mathbb{E} F\left(\eta_{0}\right)}{t \varepsilon}=0,
\end{aligned}
$$

and since $\mu$ is an invariant distribution,

$$
\begin{gathered}
\mathbb{P}\left\{\left\langle c_{g}, \eta_{0}\right\rangle>\varepsilon\right\}=\inf _{t \geq 0} \mathbb{P}\left\{\left\langle c_{g}, \eta_{t-}\right\rangle>\varepsilon\right\} \\
\leq \liminf _{M \rightarrow \infty}\left(\mathbb{P}\left\{\eta_{0}^{M} \neq \eta_{0}\right\}+\inf _{t \geq 0} \mathbb{P}\left\{\left\langle c_{g}, \eta_{t-}^{M}\right\rangle>\varepsilon\right\}\right)=0,
\end{gathered}
$$

which contradicts to $\mathbb{E}\left\langle c_{g}, \eta_{0}\right\rangle=\infty$ and therefore completes the proof of the proposition.

\section{References}

[1] F. Baccelli, F. Mathieu, and I. Norros. Mutual service processes in Euclidean spaces: existence and ergodicity. Queueing Syst., 86(1-2):95$140,2017$.

[2] R. Durrett. An infinite particle system with additive interactions. Adv. in Appl. Probab., 11(2):355-383, 1979.

[3] A. M. Etheridge and T. G. Kurtz. Genealogical constructions of population models. Ann. Probab., 47(4):1827-1910, 2019. 
[4] S. N. Ethier and T. G. Kurtz. Markov processes. Wiley Series in Probability and Mathematical Statistics: Probability and Mathematical Statistics. John Wiley \& Sons, Inc., New York, 1986. Characterization and convergence.

[5] D. Finkelshtein, Y. Kondratiev, and Y. Kozitsky. Glauber dynamics in continuum: a constructive approach to evolution of states. Discrete and Cont. Dynam. Syst. - Ser A., 33(4):1431-1450, 42013.

[6] D. Finkelshtein, Y. Kondratiev, and O. Kutoviy. Individual based model with competition in spatial ecology. SIAM J. Math. Anal., 41(1):297-317, 2009

[7] D. Finkelshtein, Y. Kondratiev, and O. Kutoviy. Semigroup approach to birth-and-death stochastic dynamics in continuum. J. Funct. Anal., 262(3):1274-1308, 2012.

[8] D. Finkelshtein, Y. Kondratiev, and O. Kutoviy. Establishment and fecundity in spatial ecological models: statistical approach and kinetic equations. Infin. Dimens. Anal. Quantum Probab. Relat. Top., 16(2):1350014, 24, 2013.

[9] D. Finkelshtein and P. Tkachov. Kesten's bound for sub-exponential densities on the real line and its multi-dimensional analogues. Advances in Applied Probability, 50(2):373-395, 2018.

[10] M. Friesen. Non-equilibrium dynamics for a Widom-Rowlinson type model with mutations. J. Stat. Phys., 166(2):317-353, 2017.

[11] M. Friesen and Y. Kondratiev. Stochastic averaging principle for spatial birth-and-death evolutions in the continuum. J. Stat. Phys., 171(5):842-877, 2018.

[12] N. Fournier and S. Méléard. A microscopic probabilistic description of a locally regulated population and macroscopic approximations. Ann. Appl. Probab., 14(4):1880-1919, 2004.

[13] N. L. Garcia. Birth and death processes as projections of higherdimensional poisson processes. Adv. in Appl. Probab., 27(4):911-930, 1995.

[14] N. L. Garcia and T. G. Kurtz. Spatial birth and death processes as solutions of stochastic equations. ALEA Lat. Am. J. Probab. Math. Stat., 1:281-303, 2006.

[15] N. Ikeda and S. Watanabe. Stochastic Differential Equations and Diffusion Processes. Nord-Holland publiching company, 1981. 
[16] Y. Kondratiev and Y. Kozitsky. Self-regulation in the Bolker-Pacala model. Appl. Math. Lett., 69:106-112, 2017.

[17] Y. Kondratiev and Y. Kozitsky. The evolution of states in a spatial population model. J. Dynam. Differential Equations, 30(1):135-173, 2018 .

[18] Y. Kondratiev, O. Kutoviy, and S. Pirogov. Correlation functions and invariant measures in continuous contact model. Infin. Dimens. Anal. Quantum Probab. Relat. Top., 11(2):231-258, 2008.

[19] Y. Kondratiev and E. Lytvynov. Glauber dynamics of continuous particle systems. Ann. Inst. H. Poincaré Probab. Statist., 41(4):685-702, 2005 .

[20] Y. Kondratiev, S. Pirogov, and E. Zhizhina. A quasispecies continuous contact model in a critical regime. J. Stat. Phys., 163(2):357-373, 2016.

[21] Y. Kondratiev and A. Skorokhod. On contact processes in continuum. Infin. Dimens. Anal. Quantum Probab. Relat. Top., 9(2):187-198, 2006.

[22] Z. Li. Measure-Valued Branching Markov Processes. Probability and Its Applications. Springer Berlin Heidelberg, Berlin, Heidelberg, 2011.

[23] T. M. Liggett. Interacting particle systems. Grundlehren der Mathematischen Wissenschaften. Springer, 1985.

[24] S. P. Meyn and R. L. Tweedie. Stability of Markovian processes. III. Foster-Lyapunov criteria for continuous-time processes. Adv. in Appl. Probab., 25(3):518-548, 1993.

[25] O. Ovaskainen, D. Finkelshtein, O. Kutoviy, S. Cornell, B. Bolker, and Y. Kondratiev. A general mathematical framework for the analysis of spatiotemporal point processes. Theoretical ecology, 7(1):101-113, 2014.

[26] X. Qi. A functional central limit theorem for spatial birth and death processes. Adv. in Appl. Probab, 40(3):759-797, 2008.

[27] J. Shi and R. Shivaji. Persistence in reaction diffusion models with weak Allee effect. Journal of Mathematical Biology, 52(6):807-829, 2006.

[28] D. Surgailis. On multiple Poisson stochastic integrals and associated Markov semigroups. Probab. Math. Statist., 3(2), 1984. 